\title{
EXPLICIT FORMS OF WICK TENSOR POWERS IN GENERAL WHITE NOISE SPACES
}

\author{
ZHIYUAN HUANG, XIAOSHAN HU, and XIANGJUN WANG
}

Received 3 April 2001 and in revised form 9 October 2001

\begin{abstract}
This paper is devoted to construction and investigation of explicit forms of Wick tensor powers in general white noise spaces. We give an extension of some objects and structure of Gaussian analysis to the case of more general white noise measures on $E^{*}$ (the dual of a nuclear space $E$ ), such that the random variable $\langle\omega, \xi\rangle$ is infinitely divisible distributed for any $\xi \in E$ and $\omega \in E^{*}$.
\end{abstract}

2000 Mathematics Subject Classification: 60H40, 46F25.

1. Introduction. White noise analysis is an important and popular theme which is intensively and extensively studied in many works (see, e.g., [4, 5, 6] and the references therein). These investigations are essentially based on the concept of Gaussian measure and the associated expansion into Hermite polynomials. Gaussian measures are remarkable objects, but in applications to some problems of mathematical physics we need to use measures which are obtained as very singular perturbations of Gaussian ones (as in quantum theory), or are constructed by the Gibbs approach (as in statistical physics).

The problem of constructing a non-Gaussian stochastic analysis is a subject of much current interest. In $[1,2,7]$ much progress has been made in non-Gaussian infinitedimensional analysis, such as Poisson white noise analysis, Gamma white noise analysis, and so forth. As we know, renormalization is a technique of extracting the principal parts of infinities. In infinite-dimensional analysis, the kernel of the chaos expansion is an important tool (e.g., the Hermite kernel, the Charlier kernel, and the Laguerre kernel). There exist many kinds of renormalization procedures in physical literatures in which the Wick calculus is crucial. Recently, the white noise analysis initiated by Hida [5] has been extensively applied to quantum physics. It provides a suitable framework for dealing with quantum stochastic calculus, and enables us to formulate the renormalization theory on sound mathematical foundation. However, the question that still remains is to find the explicit forms of Wick tensor powers in general white noise spaces. In this paper, we confine ourselves to the case of the measure $\mu$ on $E^{*}=\mathscr{D}^{*}\left(\mathbb{R}^{d}\right)$, the space of Schwartz distributions, as the dual of a nuclear space $E$ $=\mathscr{D}\left(\mathbb{R}^{d}\right)$ (i.e., the space of Schwartz test functions), such that its Laplace transform takes the form

$$
L(\xi):=\int_{E^{*}} e^{\langle\omega, \xi\rangle} \mu(d \omega)=\exp \{\langle f(\xi)\rangle\}, \quad \xi \in E,
$$


where $\langle\cdot, \cdot\rangle$ is the dual pairing of $E^{*}$ and $E,\langle f(\xi)\rangle:=\int_{\mathbb{R}^{d}} f(\xi(x)) d x$, and $\phi(t):=$ $\exp \{f(t)\}$ is the Laplace transform of some one-dimensional infinitely divisible distribution. In this case, $\mu$ is called white noise measure lifted up from this onedimensional distribution (see [3]). In the sequel, we will give a unified treatment of Wick tensor powers in this general white noise space.

2. Moments and orthogonal polynomials in one-dimensional case. Throughout this section, we consider a real random variable $X$ on a certain probability space whose all moments exist. We denote by $\phi(t)$ the Laplace transform of the probability distribution of $X$, then for $n \geq 1, \phi^{(n)}(0)$ is exactly the $n$-order moment of $X$.

For any $x \in \mathbb{R}$, let $\mathscr{P}_{0}(x) \equiv 1$. For $n \geq 1$, let $\Delta_{n}$ denote the Hankel determinant of order $n$

$$
\begin{aligned}
\Delta_{n} & =\left|\begin{array}{ccccc}
m_{0} & m_{1} & m_{2} & \cdots & m_{n-1} \\
m_{1} & m_{2} & m_{3} & \cdots & m_{n} \\
m_{2} & m_{3} & m_{4} & \cdots & m_{n+1} \\
& & & \vdots & \\
m_{n-1} & m_{n} & \cdots & \cdots & m_{2 n-2}
\end{array}\right|, \\
\mathscr{P}_{n}(x) & =\frac{1}{\Delta_{n}}\left|\begin{array}{ccccc}
m_{0} & m_{1} & m_{2} & \cdots & m_{n} \\
m_{1} & m_{2} & m_{3} & \cdots & m_{n+1} \\
& & & \vdots & \\
m_{n-1} & m_{n} & m_{n+1} & \cdots & m_{2 n-1} \\
1 & x & x^{2} & \cdots & x^{n}
\end{array}\right| .
\end{aligned}
$$

Then, $\mathscr{P}_{n}(x)$ is a polynomial of degree $n$. Furthermore, we have the following proposition.

Proposition 2.1. The polynomials $\left\{\mathscr{P}_{n}(x)\right\}_{n \geq 0}$ constitute an orthogonal system with respect to the distribution of $X$

$$
\begin{aligned}
E\left[\mathscr{P}_{n}(X) \mathscr{P}_{m}(X)\right] & =\frac{\Delta_{n+1}}{\Delta_{n}} \delta_{n m}, \quad m, n \in \mathbb{N}, \\
E\left[X^{n} \mathscr{P}_{n}(X)\right] & =\frac{\Delta_{n+1}}{\Delta_{n}}, \quad n \in \mathbb{N},
\end{aligned}
$$

where $\delta_{n m}$ is the Kronecker notation.

Proof. For any $n \geq 1, m<n$, by (2.2), we have $E\left[X^{m \mathscr{P}_{n}}(X)\right]=0$. On the other hand, noticing that $E\left[X^{n} \Delta_{n} \mathscr{P}_{n}(X)\right]$ is the coefficient of $x^{n+1}$ in the polynomial $\Delta_{n+1} \mathscr{P}_{n+1}(X)$, we immediately obtain (2.3).

Proposition 2.2. The polynomials $\left\{\mathscr{P}_{n}(x)\right\}_{n \geq 0}$ obey the following recursive formulas:

$$
\begin{gathered}
\mathscr{P}_{0}(x)=1, \\
\mathscr{P}_{1}(x)=x-m_{1}, \\
\mathscr{P}_{n+1}(x)=\left(x-c_{n}\right) \mathscr{P}_{n}(x)-\frac{\Delta_{n-1} \Delta_{n+1}}{\Delta_{n}^{2}} \mathscr{P}_{n-1}(x), \quad n \geq 1,
\end{gathered}
$$

where $c_{n}=\left(\Delta_{n} / \Delta_{n+1}\right) E\left[X \mathscr{P}_{n}^{2}(X)\right]$. 
Proof. There are constants $c_{0}, c_{1}, \ldots, c_{n}$ such that

$$
x \mathscr{P}_{n}(x)=c_{0} \mathscr{P}_{0}(x)+c_{1} \mathscr{P}_{1}(x)+\cdots+c_{n} \mathscr{P}_{n}(x)+\mathscr{P}_{n+1}(x) .
$$

So, by Proposition 2.1, we derive that $c_{0}=c_{1}=\cdots=c_{n-2}=0$ and $c_{n-1}=\Delta_{n-1} \Delta_{n+1} / \Delta_{n}^{2}$. Multiplying both sides by $\mathscr{P}_{n}(x)$ and taking expectation ( $x$ being replaced by $X$ ), we finally obtain (2.4).

Proposition 2.3. If $\varphi(t)=\exp \{f(t)\}$ is the Laplace transform of distribution of $X$, then the moments have the following forms:

$$
m_{n}=\sum_{k_{1}+k_{2}+\cdots+k_{q}=n} \frac{n !}{q !} \prod_{j=1}^{q} \frac{f^{\left(k_{j}\right)}(0)}{k_{j} !} .
$$

EXAMPLE 2.4. If $X \sim N(0,1)$, by calculation we have $m_{2 n-1}=0, m_{2 n}=(2 n-1) !$, $\Delta_{n}=(n-1) !(n-2) ! \cdots 2 ! 1 !$, and $c_{n}=0$, then

$$
\mathscr{P}_{n+1}(x)=x \mathscr{P}_{n}(x)-n \mathscr{P}_{n-1}(x) .
$$

So, $\left\{\mathscr{P}_{n}(x)\right\}_{n \geq 0}$ are Hermite polynomials $\left\{\mathscr{H}_{n}(x)\right\}_{n \geq 0}$. Its generating function is

$$
\exp \left\{t x-\frac{t^{2}}{2}\right\}=\sum_{n=0}^{\infty} \frac{t^{n}}{n !} \mathscr{H}_{n}(x) .
$$

EXAMPLE 2.5. If $X \sim \Pi(1)$ is the Poisson distribution with parameter $\lambda=1$, we have $m_{1}=1, m_{2}=2, \ldots, c_{n}=n+1, \Delta_{n}=(n-1) !(n-2) ! \cdots 2 ! 1$ !, then

$$
\mathscr{P}_{n+1}(x)=(x-n-1) \mathscr{P}_{n}-n \mathscr{P}_{n-1}(x) .
$$

Consequently, $\left\{\mathscr{P}_{n}(x)\right\}_{n \geq 0}$ are the Charlier polynomials $\left\{\mathscr{C}_{n}(x)\right\}_{n \geq 0}$, whose generating function is

$$
\exp \{x \ln (1+t)-t\}=\sum_{n=0}^{\infty} \frac{t^{n}}{n !} \mathscr{b}_{n}(x) .
$$

EXAMPLE 2.6. If $X \sim \Gamma(1,1)$ is the Gamma distribution with parameters $\lambda=1$ and $r=1$ (namely, the exponential distribution), we have $m_{n}=n !, \Delta_{n}=\Pi_{k=1}^{n-1}(k !)^{2}$, and $c_{n}=2 n+1$. Then

$$
\begin{gathered}
\mathscr{P}_{1}(x)=x-1 \\
\mathscr{P}_{2}(x)=x^{2}-4 x+2, \\
\mathscr{P}_{n+1}(x)=(x-2 n-1) \mathscr{P}_{n}(x)-n^{2} \mathscr{P}_{n-1}(x) .
\end{gathered}
$$

Namely, $\left\{\mathscr{P}_{n}(x)\right\}_{n \geq 0}$ are the Laguerre polynomials $\left\{\mathscr{L}_{n}(x)\right\}_{n \geq 0}$ whose generating function is

$$
\exp \left\{\frac{x t}{1+t}-\ln (1+t)\right\}=\sum_{n=0}^{\infty} \frac{t^{n}}{n !} \mathscr{L}_{n}(x)
$$


3. Wick tensor powers on infinite-dimensional spaces. We briefly recall some notions and notations in white noise analysis. Let $H=L^{2}\left(\mathbb{R}^{d}\right)$ be the Hilbert space of all square integrable functions defined on $\mathbb{R}^{d}$, and let $\Phi=\Gamma(H)$ be the Boson Fock space over $H$. The Schwartz spaces of $C^{\infty}$-functions with compact support on $\mathbb{R}^{d}$ and distributions will be denoted by $E$ and $E^{*}$, respectively. Then we have a Gel'fand triplet

$$
E \subset H \subset E^{*}
$$

with dual pairing $\langle\cdot, \cdot\rangle$. Suppose that the measure $\mu$ on $E^{*}$ can be defined by its Laplace transform

$$
L(\xi):=\int_{E^{*}} e^{\langle\omega, \xi\rangle} \mu(d \omega), \quad \xi \in E
$$

Then, we make the following assumptions about $\mu$ :

$$
L(\xi)=\exp \{\langle f(\xi)\rangle\}, \quad \xi \in E,
$$

where $\langle f(\xi)\rangle:=\int_{\mathbb{R}^{d}} f(\xi(x)) d x$ and $\phi(t)=\exp \{f(t)\}$ is the Laplace transform of a certain one-dimensional random variable $X$ with infinitely divisible distribution, and the generating function of the orthogonal polynomials $\mathscr{P}_{n}(x)$ with respect to $X$ is of the form

$$
G(x, t):=\sum_{n=0}^{\infty} \frac{t^{n}}{n !} \mathscr{P}_{n}(x)=\exp \{x \alpha(t)-f(\alpha(t))\},
$$

where $\alpha(0)=0$ and $\alpha(t)$ is analytic in some neighborhood of zero. Hence, $\mu$ is white noise measure lifted from this one-dimensional distribution (see [3]). Now we lift up the generating function to the infinite-dimensional space $E^{*}$, such that

$$
G(\omega, \xi)=\exp \{\langle\omega, \alpha(\xi)\rangle-\langle f(\alpha(\xi))\rangle\}=\sum_{n=0}^{\infty} \frac{1}{n !}\left\langle\mathscr{P}_{n}^{\otimes}(\omega), \xi^{\otimes n}\right\rangle,
$$

where $\mathscr{P}_{n}^{\otimes}(\omega) \in E^{* \widehat{\otimes} n}$ is called Wick tensor power. For $\omega \in E^{*}, k \geq 2$, let $\tau_{k}(\omega) \in E^{* \widehat{\otimes} k}$ be determined uniquely by the formula

$$
\left\langle\tau_{k}(\omega), \xi_{1} \hat{\otimes} \cdots \hat{\otimes} \xi_{k}\right\rangle=\left\langle\omega, \xi_{1} \cdots \xi_{k}\right\rangle,
$$

denote $\tau_{k}=\tau_{k}(1)$, then we have the following theorem.

THEOREM 3.1. The Wick tensor power has the following form:

$$
\mathscr{P}_{n}^{\otimes}(\omega)=\sum_{k_{1}+\cdots+k_{q}=n} \frac{n !}{q !} \widehat{\bigotimes}_{j=1}^{q} \frac{1}{k_{j} !}\left[\alpha^{\left(k_{j}\right)}(0) \tau_{k_{j}}(\omega)-(f \circ \alpha)^{\left(k_{j}\right)}(0) \tau_{k_{j}}\right] .
$$

Proof. Set

$$
g(t):=\langle\omega, \alpha(t \xi)\rangle-\langle f(\alpha(t \xi))\rangle
$$


Since

$$
\begin{aligned}
g^{(n)}(0) & =\alpha^{(n)}(0)\left\langle\omega, \xi^{n}\right\rangle-(f \circ \alpha)^{(n)}(0)\left\langle\xi^{n}\right\rangle \\
& =\alpha^{(n)}(0)\left\langle\tau_{n}(\omega), \xi^{\otimes n}\right\rangle-(f \circ \alpha)^{(n)}(0)\left\langle\tau_{n}, \xi^{\otimes n}\right\rangle,
\end{aligned}
$$

it follows that

$$
\left\langle\mathscr{P}_{n}^{\otimes}(\omega), \xi^{\otimes n}\right\rangle=\left.\frac{d^{n}}{d t^{n}} \exp \{g(t)\}\right|_{t=0}=\sum_{k_{1}+k_{2}+\cdots+k_{q}=n} \frac{n !}{q !} \prod_{j=1}^{q} \frac{g^{\left(k_{j}\right)}(0)}{k_{j} !},
$$

hence (3.7) holds.

Let $\mathscr{P}_{n}(\mathscr{R})$ be the space of finite linear combinations of functions of the form

$$
\omega \longmapsto\left\langle\omega, \xi_{1}\right\rangle \cdots\left\langle\omega, \xi_{n}\right\rangle=\left\langle\omega^{\otimes n}, \xi_{1} \hat{\otimes} \cdots \hat{\otimes} \xi_{n}\right\rangle, \quad \omega \in E^{*},
$$

where $\xi_{1}, \ldots, \xi_{n} \in E$. An element of the algebraic sums

$$
\mathscr{P}(\mathscr{R})=\sum_{n=0}^{\infty} \mathscr{P}_{n}(\mathscr{R})
$$

is called a polynomial.

DEFINITION 3.2. The renormalization operator $\mathscr{R}: \mathscr{P} \mapsto \mathscr{P}$ is defined by

$$
\mathscr{R}\left(\prod_{j=1}^{n}\left\langle\omega, \xi_{j}\right\rangle\right)=\left\langle\mathscr{P}_{n}^{\otimes}(\omega), \widehat{\bigotimes}_{j=1}^{n} \xi_{j}\right\rangle,
$$

and extends to a continuous linear operator on the Hida's testing functional space (E) (see [6]) which transforms the $n$-fold Stratonovich integrals into Wiener-Itô's ones, particularly,

$$
\begin{aligned}
\mathscr{R}(\exp \{\langle\omega, \xi\rangle\}) & =\sum_{n=0}^{\infty} \frac{1}{n !}\left\langle\mathscr{P}_{n}^{\otimes}(\omega), \xi^{\otimes n}\right\rangle \\
& =\exp \{\langle\omega, \alpha(\xi)\rangle-\langle f(\alpha(\xi))\rangle\}
\end{aligned}
$$

is the Wick exponential functional on the general white noise space $\left(E^{*}, \mu\right)$.

EXAMPLE 3.3 (Hermite kernel). If $X \sim N(0,1)$, then $f(t)=t^{2} / 2$,

$$
G(x, t)=\exp \left\{x t-\frac{t^{2}}{2}\right\}=\sum_{n=0}^{\infty} \frac{t^{n}}{n !} \mathscr{H}_{n}(x)
$$

hence

$$
\begin{aligned}
G(\omega, \xi) & =\exp \left\{\langle\omega, \xi\rangle-\frac{1}{2}\left\langle\xi^{2}\right\rangle\right\} \\
& =\sum_{n=0}^{\infty} \frac{1}{n !}\left\langle\mathscr{H}_{n}^{\otimes}(\omega), \xi^{\otimes n}\right\rangle .
\end{aligned}
$$


On the other hand,

$$
\begin{aligned}
\alpha(\xi) & =\xi, & & (f \circ \alpha)(\xi)=\frac{\xi^{2}}{2}, \\
\alpha^{\prime}(0) & =1, & & (f \circ \alpha)^{\prime}(0)=0, \\
\alpha^{\prime \prime}(0) & =0, & (f \circ \alpha)^{\prime \prime}(0) & =1,
\end{aligned}
$$

other derivatives vanish. For $k=0,1, \ldots,[n / 2]$, if there are $k$ of the integers $k_{1}, \ldots, k_{q}$ equal to 2 , and other $n-2 k$ integers equal to 1 , then $q=n-k$

$$
\begin{aligned}
\mathscr{H}_{n}^{\otimes}(\omega) & =\sum_{k=0}^{[n / 2]}\left(\begin{array}{c}
n-k \\
k
\end{array}\right) \frac{n !}{(n-k) ! 2^{k}}\left(-\tau_{2}\right)^{\otimes k} \hat{\otimes} \omega^{\otimes n-2 k} \\
& =\sum_{k=0}^{[n / 2]} \frac{(-1)^{k} n !}{2^{k} k !(n-2 k) !} \tau_{2}^{\otimes k} \hat{\otimes} \omega^{\otimes n-2 k} .
\end{aligned}
$$

In this case we have

$$
\begin{gathered}
\mathscr{R}\left(\langle\omega, \xi\rangle^{n}\right)=\left\langle\mathscr{H}_{n}^{\otimes}(\omega), \xi^{\otimes n}\right\rangle=\sum_{k=0}^{[n / 2]} \frac{(-1)^{k} n !\|\xi\|^{2 k}\langle\omega, \xi\rangle^{n-2 k}}{2^{k} k !(n-2 k) !}, \\
\mathscr{R}\left(\prod_{j=1}^{n}\left\langle\omega, \xi_{j}\right\rangle\right)=\sum_{k=0}^{[n / 2]}(-1)^{k} \sum_{|S|=2 k}\left\langle\xi_{1}, \ldots, \xi_{n}\right\rangle_{S} \prod_{j \notin S}\left\langle\omega, \xi_{j}\right\rangle,
\end{gathered}
$$

where $S \subset\{1, \ldots, n\},|S|$ is the cardinal of $S$, and

$$
\left\langle\xi_{1}, \ldots, \xi_{n}\right\rangle_{S}=\sum\left\langle\xi_{i_{1}}, \xi_{j_{1}}\right\rangle \cdots\left\langle\xi_{i_{k}}, \xi_{j_{k}}\right\rangle
$$

where the pairs $\left\{i_{1}, j_{1}\right\}, \ldots,\left\{i_{k}, j_{k}\right\}$ run over $S$.

EXAMPLE 3.4 (Charlier kernel). If $X \sim \Pi(1)$, then $f(t)=e^{t}-1$,

$$
G(x, t)=\exp \{x \ln (1+t)-t\}=\sum_{n=0}^{\infty} \frac{t^{n}}{n !} \mathscr{C}_{n}(x),
$$

hence

$$
G(\omega, \xi)=\exp \{\langle\omega, \ln (1+\xi)\rangle-\langle\xi\rangle\}=\sum_{n=0}^{\infty} \frac{1}{n !}\left\langle\mathscr{b}_{n}^{\otimes}(\omega), \xi^{\otimes n}\right\rangle
$$

In this case, we have $\alpha(\xi)=\ln (1+\xi),(f \circ \alpha)(\xi)=\xi, \alpha^{\prime}(0)=1,(f \circ \alpha)^{\prime}(0)=1$, and for $k \geq 2, \alpha^{(k)}(0)=(-1)^{k-1}(k-1) !,(f \circ \alpha)^{(k)}(0)=0$, then

$$
\mathscr{C}_{n}^{\otimes}(\omega)=\sum_{k_{1}+\cdots+k_{q}=n} \frac{(-1)^{n-q} n !}{q !} \widehat{\bigotimes}_{j=1}^{q} \frac{1}{k_{j}} \tau_{k_{j}}(\omega),
$$


where $\tau_{1}(\omega)$ is understood as $\omega-\tau_{1}$. Moreover, in the case of $k_{j}=1,\left\langle\omega, \xi^{k_{j}}\right\rangle$ in the following expression should be replaced by $\langle\omega, \xi\rangle-\langle\xi\rangle$ :

$$
\begin{aligned}
\mathscr{R}\left(\langle\omega, \xi\rangle^{n}\right) & =\left\langle\mathscr{C}_{n}^{\otimes}(\omega), \xi^{\otimes n}\right\rangle \\
& =\sum_{k_{1}+\cdots+k_{q}=n} \frac{(-1)^{n-q} n !}{q ! k_{1} \cdots k_{q}}\left\langle\omega, \xi^{k_{1}}\right\rangle \cdots\left\langle\omega, \xi^{k_{q}}\right\rangle .
\end{aligned}
$$

EXAMPLE 3.5 (Laguerre kernel). If $X \sim \Gamma(1,1)$, then $f(t)=-\ln (1-t)$,

$$
G(x, t)=\exp \left\{x t(1+t)^{-1}-\ln (1+t)\right\}=\sum_{n=0}^{\infty} \frac{t^{n}}{n !} \mathscr{L}_{n}(x),
$$

hence

$$
G(\omega, \xi)=\exp \left\{\left\langle\omega, \xi(1+\xi)^{-1}\right\rangle-\langle\ln (1+\xi)\rangle\right\}=\sum_{n=0}^{\infty} \frac{1}{n !}\left\langle\mathscr{L}_{n}^{\otimes}(\omega), \xi^{\otimes n}\right\rangle .
$$

In this case, we have $\alpha(\xi)=\xi(1+\xi)^{-1},(f \circ \alpha)(\xi)=\ln (1+\xi)$, and for $k \geq 1, \alpha^{(k)}(0)=$ $(-1)^{k-1} k !,(f \circ \alpha)^{(k)}(0)=(-1)^{k-1}(k-1)$ !. It follows that

$$
\mathscr{L}_{n}^{\otimes}(\omega)=\sum_{k_{1}+\cdots+k_{q}=n} \frac{(-1)^{n-q} n !}{q !} \widehat{\bigotimes}_{j=1}^{q} \frac{1}{k_{j}} \tilde{\tau}_{k_{j}}(\omega),
$$

where $\tilde{\tau}_{1}(\omega)=\omega-\tau_{1}, \tilde{\tau}_{k}(\omega)=k \tau_{k}(\omega)-\tau_{k}$, for $k \geq 2$. Finally we have

$$
\begin{aligned}
\mathscr{R}\left(\langle\omega, \xi\rangle^{n}\right) & =\left\langle\mathscr{L}_{n}^{\otimes}(\omega), \xi^{\otimes n}\right\rangle \\
& =\sum_{k_{1}+\cdots+k_{q}=n} \frac{(-1)^{n-q} n !}{q !} \prod_{j=1}^{q}\left[\left\langle\omega, \xi^{k_{j}}\right\rangle-\frac{\left\langle\xi^{k_{j}}\right\rangle}{k_{j}}\right] .
\end{aligned}
$$

ACKNOWLEDGMENTS. This work was supported by the National Natural Science Foundation of China (NSFC), project 19631030 and by the Research Fund for the Doctoral Program of Higher Education (RFDP) of China, project 1999048723.

\section{REFERENCES}

[1] S. Albeverio, Yu. L. Daletsky, Yu. G. Kondratiev, and L. Streit, Non-Gaussian infinitedimensional analysis, J. Funct. Anal. 138 (1996), no. 2, 311-350.

[2] S. Albeverio, Yu. G. Kondratiev, and L. Streit, How to generalize white noise analysis to nonGaussian measures, Dynamics of Complex and Irregular Systems (Bielefeld, 1991), Bielefeld Encounters in Mathematics and Physics, vol. VIII, World Scientific Publishing, New Jersey, 1993, pp. 120-130.

[3] I. M. Gel'fand and N. Ya. Vilenkin, Generalized Functions. Vol. 4: Applications of Harmonic Analysis, Academic Press, New York, 1964.

[4] T. Hida, Brownian Motion, Applications of Mathematics, vol. 11, Springer-Verlag, New York, 1980.

[5] T. Hida, H.-H. Kuo, J. Potthoff, and L. Streit, White Noise. An Infinite-Dimensional Calculus, Mathematics and Its Applications, vol. 253, Kluwer Academic Publishers, Dordrecht, 1993. 
[6] Z.-Y. Huang and J.-A. Yan, Introduction to Infinite Dimensional Stochastic Analysis, Mathematics and Its Applications, vol. 502, Kluwer Academic Publishers, Dordrecht, 2000.

[7] E. W. Lytvynov, A note on spaces of test and generalized functions of Poisson white noise, Hiroshima Math. J. 28 (1998), no. 3, 463-480.

Zhiyuan Huang: Huazhong University of Science And Technology, Wuhan, Hubei 430074, CHINA

E-mail address: zyhuang@hust.edu.cn

XiaOshan Hu AND XianguUn Wang: HuaZhong University of SCIENCE AND TeChNOlogy, WUHAN, Hubei 430074, CHINA 


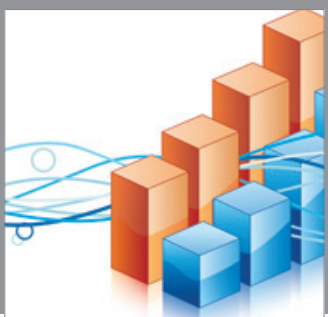

Advances in

Operations Research

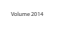

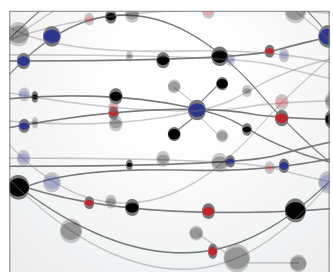

\section{The Scientific} World Journal
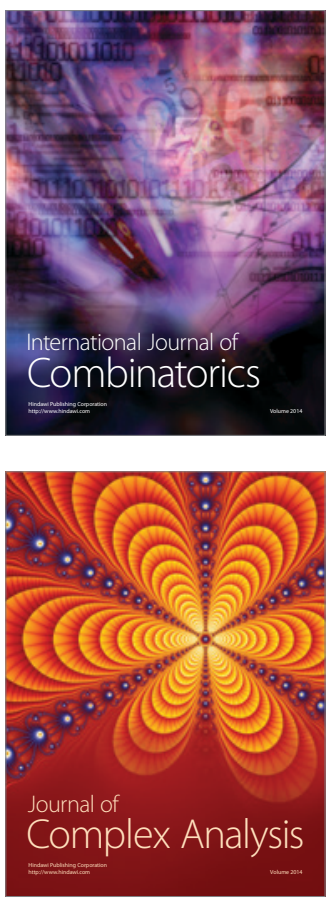

International Journal of

Mathematics and

Mathematical

Sciences
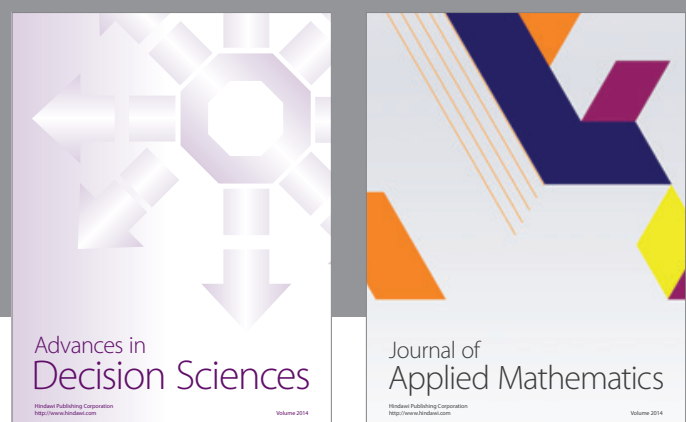

Journal of

Applied Mathematics
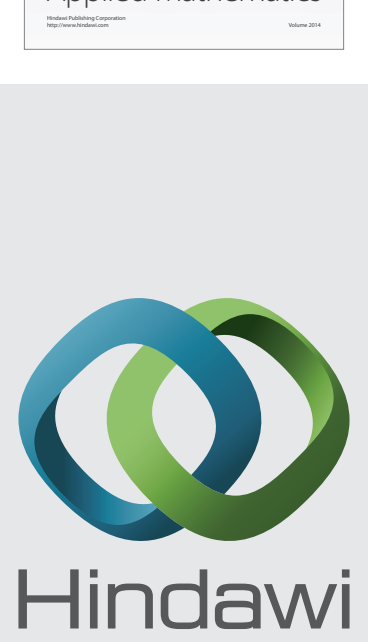

Submit your manuscripts at http://www.hindawi.com
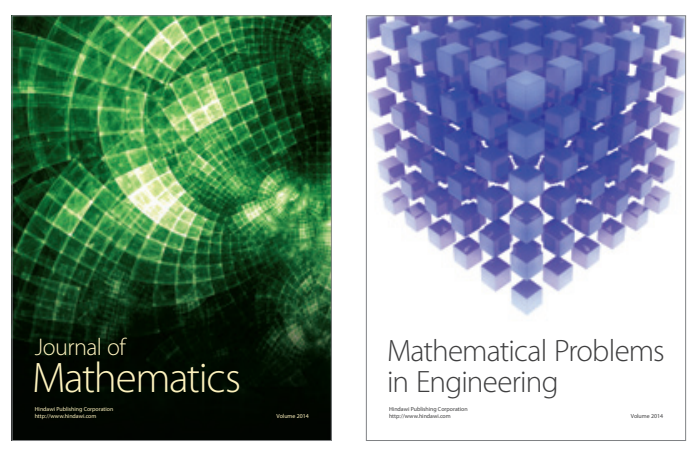

Mathematical Problems in Engineering
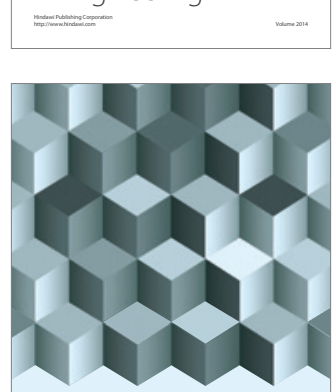

Journal of

Function Spaces
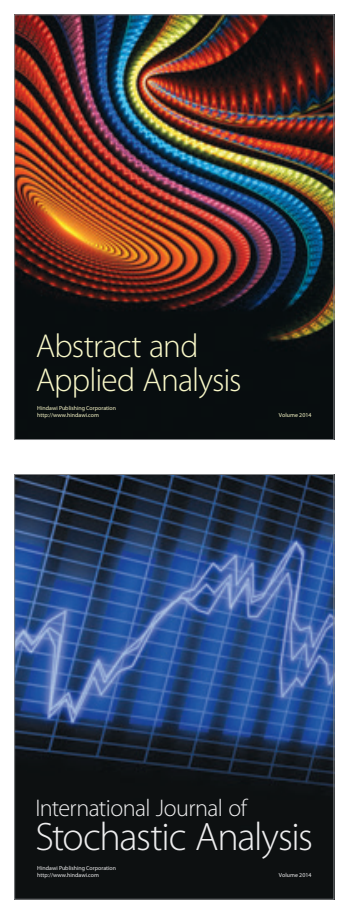

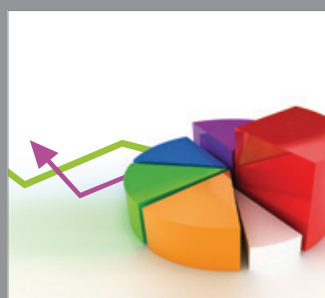

ournal of

Probability and Statistics

Promensencen
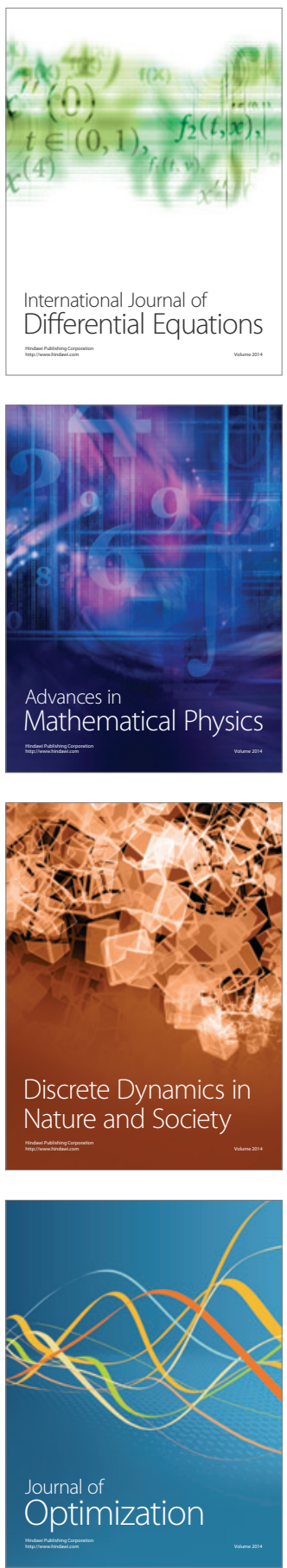